\title{
El lenguaje docente como una forma de exclusión educativa en las aulas universitarias
}

\author{
The Teacher's Language as a Way of Educational \\ Exclusion in College Classrooms
}

\author{
A linguagem docente como uma forma de exclusão educativa
}

nas salas de aula universitárias

\author{
Florlenis Chévez Ponce ${ }^{1}$ \\ Martha Patricia Astudillo Torres ${ }^{2}$ \\ Álvaro Artavia Medrano 3
}

\section{Resumen}

El artículo presenta la opinión que tienen los estudiantes de la Licenciatura en la Enseñanza de los Estudios Sociales y la Educación Cívica de la Universidad de Costa Rica sobre el lenguaje que utiliza el profesorado en los diferentes cursos que reciben. Se utilizó la técnica de grupos de discusión con el fin de resaltar y contrastar las diversas opiniones del estudiantado en relación con las formas de comunicación percibidas en la cotidianidad del ambiente del aula. A partir de los hallazgos, se plantea la necesidad de que el personal docente reflexione acerca del lenguaje que emplea, de manera que permita una participación del estudiantado con mayor libertad en la interacción propia del quehacer cotidiano del aula.

\section{Palabras clave}

proceso comunicativo; interacción en el aula; grupo de discusión; factor de exclusión

\section{Abstract}

This article presents the opinion of the students enrolled in the Social Studies Teaching and Civics Teaching majors at the University of Costa Rica, in relation to the language their professors employ in the different courses these students take. The discussion groups technique was used with the intention of exalting and contrasting the varied opinions the students have in regards to the ways of communication employed and how they are perceived in the everyday environment in the classroom. Based on the findings, the faculty's necessity to reflect on the language they use is presented, in such a way that students get to participate more freely in their own interaction while doing their in-class everyday tasks.

\section{Keywords}

communicative process; interaction in the classroom; discussion group; exclusion factor

\section{Resumo}

0 artigo apresenta a opinião dos estudantes da Licenciatura em Ensino dos Estudos Sociais e a Educação Cívica da universidade de Costa Rica sobre a linguagem utilizada pelo professorado nas diferentes aulas que eles têm. Utilizou-se a técnica de grupos de discussão com o fim de ressaltar e contrastar as diversas opiniões dados estudantes em relação às formas de comunicação percebidas no dia a dia no ambiente da aula. A partir das descobertas, é preciso que os professores reflitam acerca da linguagem que utilizam para permitir uma participação dos estudantes com maior liberdade na interação própria das atividades cotidianas na sala de aula.

\section{Palavras chave}

processo comunicativo; interação na sala de aula; grupo de discussão; fator de exclusão

Artículo recibido el 20 de abril del 2016 y aprobado el 21 de diciembre del 2016

1 Universidad de Costa Rica, San José, Costa Rica. Correo electrónico: florlenis.chevez.ponce@mep.go.cr

2 Universidad Autónoma de Chiapas, Tuxtla Gutiérrez, Chiapas, México. Correo electrónico: patricia.astudillo@unach.me

3 Universidad de Costa Rica, San José, Costa Rica. Correo electrónico: alvartavia@mep.go.cr 


\section{Introducción}

El lenguaje docente es uno de los aspectos que tiene diariamente un papel fundamental en la comunicación que se lleva a cabo en las clases que se imparten en los centros educativos. Por ello, es preciso considerar la percepción del estudiantado con respecto al lenguaje que el personal docente emplea como uno de los componentes de los procesos de enseñanza y aprendizaje.

Para lograr recabar evidencias acerca de la percepción con respecto al proceso de comunicación que se lleva a cabo en diferentes cursos universitarios, se contó con la participación de estudiantes de la Licenciatura en la Enseñanza de los Estudios Sociales y Educación Cívica de la Universidad de Costa Rica. Con estos alumnos se desarrolló un grupo de discusión, técnica seleccionada con el fin tanto de reflejar preocupaciones de las personas involucradas, como de recuperar las voces individuales dentro de los debates que se susciten (Barbour, 2013).

Este trabajo se enmarca en una conceptualización tanto del proceso comunicativo (Escandell, 2005), como de los aspectos que intervienen en el lenguaje (Van Dijk, 2002). Posteriormente, se detallan aspectos metodológicos y los hallazgos propios de la investigación, y una serie de consideraciones finales a partir de las evidencias con el propósito de contribuir al campo educativo.

\section{Referente teórico}

El referente teórico para fundamentar este trabajo lo conforman dos elementos fundamentales: por un lado, el proceso de la comunicación que expone Escandell (2005) desde una perspectiva diferente a la clásica; por otro lado, la conceptualización de lenguaje de Van Dijk $(2001,2002)$ y algunos elementos que tienen relación en la comunicación del aula. Dichos elementos teóricos conforman la base para complementar la integración de las categorías y el posterior análisis de la información.

\section{Comunicación}

Tradicionalmente, la comunicación se ha concebido como la relación que se manifiesta en la interacción de cuatro elementos del modelo clásico comunicativo: emisor, receptor, mensaje y contexto. No obstante, este proceso no es claro en cuanto a reflejar las diferencias existentes entre cada elemento, ni confiere a cada componente el lugar que le corresponde.

En contraposición con el modelo clásico de comunicación, Escandell (2005) distingue tres categorías: (1) los elementos, que son las entidades físicas básicas, es decir, los componentes directamente observables en un acto de comunicación; (2) las representaciones, que son las entidades mentales que desempeñan algún tipo de papel en la comunicación, ya sea como material de entrada o como material contextual; y (3) los procesos, que son los diferentes tipos de operaciones que intervienen en el tratamiento de los datos.

Dada la relevancia de los procesos de comunicación en el desarrollo de las lecciones, los planteamientos de Escandell (2005) resultan más pertinentes, puesto que resaltan el carácter emergente del lenguaje como elemento fundamental en la relación social que se da entre enseñar y aprender. En este sentido, los procesos de comunicación juegan un papel relevante en el cual todos los involucrados deben tomar una posición con respecto a lo que se dice en las aulas y cómo se dice. Por consiguiente, es indispensable que el personal docente reflexione en torno a estos componentes, de manera que el lenguaje que empleen incluya estos elementos y que la comunicación no sea un factor que influya en la exclusión educativa.

\section{Conceptualización del lenguaje}

Según Van Dijk (2002), en la definición del término lenguaje intervienen dos aspectos fundamentales: en primer término, una forma específica del uso del lenguaje; en segundo término, una forma específica de interacción social. Por ello, el lenguaje se concibe como un evento comunicativo completo en una situación social. Desde esta perspectiva, el significado del lenguaje es una estructura cognitiva, por lo que es preciso incluir en el concepto de lenguaje no solo elementos observables verbales y no verbales, o interacciones sociales y actos de 
habla, sino también las representaciones cognitivas y estrategias involucradas durante la producción o comprensión del lenguaje; es decir, interesa observar el lenguaje como un factor dinámico de las interacciones sociales, y la esquematización o normas identificables que permitan encontrar modelos para su interpretación y análisis.

\section{El lenguaje docente como forma de exclusión}

Como premisa fundamental de este trabajo, se concibe el lenguaje como un fenómeno práctico, social y cultural, así quienes hacen uso del lenguaje realizan actos sociales y participan en la interacción social, típicamente en la conversación y en otras formas de diálogo (Van Dijk, 2001). En consecuencia, para analizar si el lenguaje del personal docente actúa como un elemento de exclusión, se parte de la comunicación que se establece a través del diálogo entre estudiantes y docentes para conformar un esquema de análisis que permita identificar e interpretar las formas de intervención verbal que se originan en los procesos de enseñanza y aprendizaje.

En el ámbito educativo es relevante considerar las cuatro dimensiones que propone este autor, pues en su esencia tienen elementos que son de interés en la concepción de lenguaje como una posible forma de exclusión educativa. La primera dimensión la constituye la acción, la cual va dirigida a la clase de cosas que las personas realizan.

La segunda es el contexto, en el cual se pueden involucrar parámetros como los participantes, sus roles y propósitos, además de un marco como el tiempo y el lugar. En esta dimensión, las personas participantes o involucradas en el proceso son elementos cruciales del contexto y por ello se consideran características como ser hablante o receptor, ser hombre o mujer, ser joven o adulto, tener autoridad o prestigio, por ejemplo. Todos estos componentes pueden influenciar la producción o interpretación del texto y el habla.

Una tercera dimensión la constituye el poder, en el cual se evidencian algunos aspectos que pueden ser considerados elementos de exclusión, como el lenguaje sexista, el poder de los blancos en el texto, y el habla racista o las formas de contrapoder de algunos grupos. En esta posición, se observa cómo existe un vínculo entre el poder y el lenguaje. De esta manera, en las aulas universitarias, cuando se solicita o sugiere la realización de determinada acción, aun sin amenazas explícitas, el poder de los docentes consiste en que el estudiantado tenderá a obedecer para evitar las consecuencias negativas.

La cuarta dimensión propuesta por este autor es la ideología, que sirve de interfaz entre los intereses colectivos del grupo y las prácticas sociales individuales. Así, las ideologías son las representaciones mentales que forman la base de la cognición social, además de una función social de coordinación, por lo que tienen funciones organizativas de las creencias que se reflejan en un nivel muy general de pensamiento, les dicen a las personas cuál es su posición y qué deben pensar acerca de las relaciones sociales.

El planteamiento de estas dimensiones permite reflexionar sobre cuáles características pueden influir en un lenguaje docente que se concrete en la práctica con carga de exclusión educativa en las aulas universitarias.

\section{Metodología}

Dada la naturaleza del fenómeno por estudiar, se debía emplear una técnica que permitiera la reconstrucción del sentido socioeducativo en el seno de una situación discursiva (Gurdián, 2010), por lo que se trabajó con un grupo de discusión. La técnica y otras consideraciones metodológicas se exponen a continuación.

\section{Conceptualización de la técnica}

Los grupos de discusión son una técnica orientada a la captura de material de la vida real en ambientes sociales. Estos se utilizan para "profundizar en expectativas, conocimiento, opiniones e ideologías expresadas por las personas que tienen que ver o que están directamente involucradas en la realidad socioeducativa que queremos investigar" (Gurdián, 2010, p. 210). En ocasiones, los grupos de discusión actúan como foros en los que las personas se preocupan por un tema específico, por lo que también suelen ser ayuda para formular soluciones 
potenciales, aunque no sea inicialmente su propósito (Barbour, 2013).

La unidad principal de análisis en la investigación con grupos de discusión es el grupo mismo, por lo que su conformación debe partir del hecho de que las personas que lo integran compartan al menos una característica importante, por ejemplo, homogeneidad en su origen, lo cual no se trata en modo alguno de que sus actitudes y opiniones sean similares.

Las acciones por seguir en el planteamiento y desarrollo de grupos de discusión, según Hernández, Fernández y Baptista (2010), son:

- Determinar un número provisional de grupos y sesiones.

- Definir el perfil de quienes participarán.

- Detectar personas del tipo elegido.

- Invitación a las sesiones.

- Organización de las sesiones.

- Llevar a cabo la sesión.

- Elaboración del reporte de la sesión.

Dos aspectos sumamente relevantes en el desarrollo de esta técnica son la elaboración de una guía temática, y el papel de la persona que investiga y que actúa como moderadora en la discusión, sobre todo porque con estos dos elementos es que se logran abarcar las ideas sobre las construcciones sociales, contradicciones, distinciones o salvedades que las personas participantes plantean sobre la marcha.

\section{Selección de participantes}

La composición de un grupo para el desarrollo de esta técnica es una decisión en la que confluyen diversos aspectos, como metodología, estrategias y ética. Por una parte, se espera reflejar la diversidad dentro del sector o población que se estudia, aunque también se parte de la necesidad de que una selección deliberada permita el examen cuidadoso de los datos para llevar a cabo una comparación sistemática. Finalmente, se hace necesaria la consideración de la facilidad con que se puede dar el turno de hablar a las personas que integran el grupo, lo cual, a su vez, resulta una habilidad de la persona que modera y de la complejidad deseada para el debate.

En este estudio se elaboró un perfil que permitiera seleccionar, a partir de ciertos criterios, a las personas participantes, los cuales son:

- Cursar actualmente la carrera de Licenciatura en la Enseñanza de los Estudios Sociales y Educación Cívica en la Universidad de Costa Rica.

- Haber aprobado cursos en las diferentes escuelas de las cuales forma parte esta carrera (formación docente, historia, geografía, por ejemplo).

- Haber desarrollado su práctica docente (experiencia académico-laboral en una institución de educación media o secundaria).

Estos criterios o características básicas para la selección de participantes permitieron la búsqueda de personas que cumplieran tales requisitos, para lo que fue necesaria la colaboración administrativa de la Escuela de Formación Docente, unidad académica en la que se encuentra la carrera mencionada. La especificidad tanto de la carrera como de los otros dos criterios fue una decisión de los investigadores debido al perfil académico propuesto en el plan de estudios de quienes se egresan, y porque los diferentes cursos que llevan proponen la formación de personas críticas y conscientes de la realidad que les circunda.

De igual modo, se consideró vital el haber concluido la práctica docente como una forma de aproximación de cada persona al aula ya no como estudiante, sino en una labor docente, en la que también pudiera cuestionarse los procesos de comunicación en los que participa, ahora desde nuevas perspectivas.

\section{Aplicación de la técnica}

Un aspecto clave en el desarrollo de la técnica de grupos de discusión es la guía temática, la cual intenta anticipar el debate e imaginar posibles respuestas a la conversación o al debate que se suscite (Barbour, 2013). 
La guía temática fue elaborada tomando como punto de partida tres preguntas generadoras de interacción y debate en las personas participantes:

- ¿Cuáles ejemplos pueden dar de la utilización que el personal docente hace del lenguaje en el desarrollo de las lecciones, que van desde mensajes escritos, gestuales y verbales hasta una clara estructura discursiva?

- ¿Cuáles elementos del contexto intervienen en el proceso comunicativo entre docentes y estudiantes que dan cuenta del conocimiento mutuo y el saber compartido?

- ¿Qué relaciones o vínculos internos entre docentes y estudiantes se evidencian en el desarrollo de contenidos y actividades en las clases de los cursos que han llevado a lo largo de su carrera?

Como material de estímulo para el debate, se empleó un corto video en el que se reflejaba un fragmento del desarrollo de una lección.

Dado que el efecto que ejerce sobre el grupo la persona que investiga y modera la discusión, se debe tomar este asunto como relevante por las implicaciones tanto éticas como metodológicas en la presentación de los hallazgos. Así mismo, Calderón y Alzamora (2008) señalan como elemento esencial las condiciones de aplicación; consiguientemente, se debe insistir en la necesidad de que cada persona participante utilice sus propios conocimientos, experiencias y lenguaje. Por esta razón, resulta conveniente explicar tanto el propósito de la técnica, como el contenido y los objetivos de cada una de las preguntas generadoras de discusión.

En congruencia con los anteriores planteamientos, se conformaron tres grupos de trabajo con las personas seleccionadas. Una de las sesiones se llevó a cabo en la sala de trabajo del Programa de Doctorado en Educación de la Universidad de Costa Rica y las otras dos sesiones se realizaron en un sitio destinado a oficinas de docentes para la atención a estudiantes. El tiempo de duración de las sesiones fue de una hora y treinta minutos cada una.

\section{Presentación de los hallazgos}

Las sesiones en las que se desarrolló la técnica permitieron contar con un cúmulo de hallazgos que requiere de una categorización para su análisis. Para ello, se partió del respaldo teórico y de las evidencias obtenidas con la técnica para establecer las tres categorías que se muestran en la tabla 1.

Tabla 1. Categorías de análisis ${ }^{4}$

\section{Categorias}

Lenguaje: es una herramienta educativa que puede promover el desarrollo intelectual, emocional, moral y social del educando mediante las expresiones utilizadas en el desarrollo de los contenidos de las diferentes materias durante las lecciones.

Relación de igualdad entre los interlocutores: se refiere a los factores contextuales que intervienen en el proceso comunicativo entre los principales actores del proceso de enseñanza y aprendizaje.

\section{Elementos}

Formas verbales sexistas
Estructura discursiva
Presencia de términos abstractos
Diálogo
Tema punto de vista científico o profesional
Mensajes: verbales, escritos y gestuales
Elementos observables y no observables
Conocimiento mutuo
Saber compartido
Vínculos de respeto, aprecio y tolerancia
Origen étnico del estudiante
Procedencia geográfica del estudiante

4 Discurso docente: en el discurso docente se describen los parámetros comunicativos que condicionan las características lingüísticas de ambos tipos de discurso: oral y escrito. En sentido restringido, se puede considerar el discurso como un conjunto de palabras y frases utilizadas para manifestar lo que se piensa o siente. El entramado lingüístico permite expresar ideas, opiniones y estados afectivos en orden de facilitar el proceso de enseñanza y aprendizaje. 


\begin{tabular}{l|l}
\multicolumn{1}{c|}{ Categorías } & \\
\hline $\begin{array}{l}\text { Interacción en el aula: la interacción está presente } \\
\text { como ejemplo más destacado de la trascendencia de }\end{array}$ & Ámbito de la cultura \\
\hline $\begin{array}{l}\text { la comunicación en el proceso de enseñanza. A todo } \\
\text { ello se une el hecho de que incluso durante el periodo } \\
\text { de presentación de los contenidos o prácticas, existe }\end{array}$ & Espacio y tiempo compartido \\
\hline una relación entre el profesorado y el estudiantado. & Construcción de la disertación compartida \\
\hline & Diferencia jerárquica social o funcional \\
& Vínculos internos \\
\hline & Participación \\
\hline
\end{tabular}

Según lo indicado por los estudiantes que participaron en el grupo de discusión, la información obtenida se agrupó en cada categoría de la siguiente manera:

- Lenguaje docente: aquí se concentró la información sobre todo lo que se relaciona con la expresión propia del personal docente, principalmente el uso del lenguaje en el desarrollo de las temáticas que se abordan en las clases.

- Relación de igualdad entre interlocutores: en esta categoría se agrupó la información sobre los factores contextuales que influyen en el proceso de comunicación en las clases.

- Interacción en el aula: en esta categoría se agrupó todo lo relacionado con las relaciones que se manifiestan entre estudiantes y docentes en el aula.

En cuanto a la primera categoría, el lenguaje docente, las personas participantes manifestaron que hay ciertos profesores que realizaban comentarios con los que deslegitimaban o se mofaban de la opinión de compañeros(as). Por otra parte, indicaron que frecuentemente la rigidez del personal docente impide opinar respecto de una temática, pues sienten temor a que se haga referencia a su intervención de manera negativa.

Uno de los aspectos de mayor relevancia fue la mención de las diferencias que el personal docente hace tomando como referencia el origen étnico del estudiantado, o bien, la percepción de rasgos o características físicas. Un ejemplo de esto es el siguiente:

Yo recuerdo que también otra experiencia de eso fue en un curso de historia y que teníamos una compañera que tenía facciones achinaditas; estábamos hablando del componente indígena y cuestiones así y específicamente de la problemática que viven los poblados indígenas, entonces la profesora vuelve a ver a la compañera y le dice que explique su experiencia, como si la compañera por tener esas facciones fuera indígena; entonces, en ese momento la compañera, que era bastante tímida, se puso colorada, bueno hasta cierto punto incómoda y dijo que nada más tenía facciones pero que no conocía cuáles eran esas problemáticas. (M., comunicación personal, 16 julio, 2013).

Además, expusieron que otro factor que consideraban excluyente es la utilización de un vocabulario muy propio del área metropolitana, en detrimento del estudiantado que proviene de áreas rurales o del extranjero, lo cual provoca su desubicación y falta de comprensión en cuanto a las explicaciones dadas por docentes; el estudiante refiere: "Muchas veces los docentes no toman en cuenta este aspecto y hacen alusión a ejemplos y palabras de un contexto al que uno no está acostumbrado. Eso desmotiva, ya que no se entiende y no se puede participar" (S., comunicación personal, 17 julio 2013).

En relación con la segunda categoría, específicamente en lo que se refiere a vínculos de respeto y tolerancia como factores contextuales en la comunicación, se comentó que: "Hay profesores que no concuerdan en lo que dicen con lo que hacen, ¿verdad?, hablan de respeto hablan de tolerancia y pues uno ve faltándole el respeto de una manera directa a otros estudiantes" (C., comunicación personal, 18 julio 2013).

En esta misma línea abundaron comentarios en los que se manifestaban evidencias de ciertos rasgos de exclusión educativa en las clases que han recibido. Con respecto a lo que plantea Van Dijk (2002), si se controla el contexto también se puede controlar gran 
parte de las estructuras del lenguaje; por su parte, los usuarios del lenguaje deben tener claro que no solamente forman modelos mentales de los eventos sobre los que hablan, sino que también forman modelos de los eventos en los cuales participan, por ello en la comunicación que se muestra en las lecciones estos elementos adquieren relevancia.

Finalmente, en cuanto a la tercera categoría, las personas participantes señalaron la relevancia de ciertos elementos que deben generarse en los procesos de comunicación que se visualizan en las aulas, de manera que en las interacciones entre quienes forman parte del hecho comunicativo todos puedan sentirse partícipes de dicho proceso.

Los participantes manifestaron que en la carrera de Estudios Sociales tienen que llevar las asignaturas de Historia, Geografía y Educación. En el caso de los cursos de Historia y Geografía, que en su mayoría se da en forma magistral, existe poca comunicación entre los estudiantes, y entre los estudiantes y el docente. Así mismo, indicaron que en algunos cursos de Formación Docente se imparten clases con un estilo más horizontal en la comunicación. La falta de horizontalidad en la interacción en el aula a la que se hizo mención en la discusión grupal genera una brecha o división entre estudiantes y docentes, pues frecuentemente se limita la participación por el temor que suscita la diferencia jerárquica que queda manifiesta en comentarios o gestos.

\section{Consideraciones finales}

Van Dijk (2001, p. 38) señala que "los lenguajes pueden estar condicionados por los contextos, pero también ejercen influencia sobre ellos y los construyen. Esto es, los lenguajes son una parte estructural de sus contextos, y sus estructuras respectivas se influyen mutua y continuamente". Por consiguiente, el personal docente debe reflexionar acerca del lenguaje que utiliza para comunicarse con sus estudiantes, de manera que las expresiones que se concretan en el ambiente de aula permitan una participación con mayor libertad y con menor influencia de forma negativa como factor de exclusión en los procesos de enseñanza y aprendizaje.
Los hallazgos generados con la técnica permitieron identificar la percepción de desigualdades con respecto al lenguaje que utiliza el profesorado de la Licenciatura en la Enseñanza de los Estudios Sociales y la Educación Cívica. Cabe destacar que dicha carrera está integrada por docentes de tres escuelas diferentes; no obstante, las diferencias y desigualdades pareciera que se producen e incluso se reproducen por el accionar didáctico o la metodología empleada en el desarrollo de las lecciones.

En este sentido, indican que por la practicidad que se da en las clases del área de Geografía los profesores son menos "rigurosos", en tanto que en las clases de Historia se nota un predominio o exclusividad en la participación del personal docente, quienes atribuyen esto a la cantidad de contenido por abarcar en las lecciones; sin embargo, el estudiantado lo percibe como una relación de poder en la que prácticamente se impide cualquier forma de participación.

En cuanto al tercer componente de su plan de estudios - los cursos de Formación Docente-, se evidencian dos particularidades: por una parte, las personas participantes exteriorizaron que al existir muchas actividades en ocasiones no se da libertad de opinar a todo el grupo o las participaciones no se amplían; y, por otra parte, se evidencia que al haber profesores que tienen formación en enseñanza de primaria, las clases las dan con un enfoque desde esta perspectiva, por lo cual las personas que conformaron los grupos de discusión perciben exclusión educativa, debido al lenguaje y a las actividades que se llevan a cabo y que sienten lejanas a su accionar y su proceso de formación profesional inicial.

Fundamentalmente, estas manifestaciones son evidencias que deben profundizarse en otras investigaciones, en las que se considere el lenguaje empleado por el personal docente y su vinculación con la metodología, rasgos que han salido a la luz en este estudio como fuentes de exclusión educativa.

Este estudio brinda un aporte esencial al campo educativo y radica en la posibilidad de trabajar con estudiantes de otras carreras utilizando esta misma técnica, de tal modo que se pongan de manifiesto diferencias y similitudes en el lenguaje docente empleado en las aulas universitarias y su consideración por parte 
del estudiantado, a quienes no siempre se les da la posibilidad de reflexionar acerca de la comunicación que se genera en las lecciones, aspecto considerado vital sobre todo en profesionales que dedicarán su vida a la docencia.

\section{Referencias}

Barbour, R. (2013). Los grupos de discusión en investigación cualitativa. Madrid: Ediciones Morata.

Calderón Saldaña, J. y Alzamora de los Godos Urcia, L. (2008). La técnica de recolección de información mediante los grupos focales. Revista Salud, Sexualidad y Sociedad. Recuperado de: http://www. inppares.org/revistasss/Revista
Escandell, M. V. (2005). La comunicación. Madrid: Editorial Gredos, S.A.

Gurdián, A. (2010). El paradigma cualitativo en la investigación socioeducativa. San José: Editorial Universidad de Costa Rica.

Hernández Sampieri, R., Fernández Collado, C. y Baptista Lucio, P. (2010). Metodología de la investigación. México: McGraw-Hill.

Van Dijk, T. (2001). El lenguaje como interacción social. Estudios sobre el lenguaje II. Una introducción multidisciplinaria. Barcelona: Gedisa Editorial.

Van Dijk, T. (2002). Conocimiento, elaboración del lenguaje y educación. Manizales: Escribanía.

Para citar este artículo

Artavia, A., Astudillo, M., y Chévez, F. (2017). El lenguaje docente como una forma de exclusión educativa en las aulas universitarias. Folios, (46), 97-104. 